http://dx.doi.org/10.21611/qirt.1994.013

\title{
A procedure to measure thermal conductivities of anis otropic laminates by infrared thermography
}

\author{
by BERARDI P.G.* and CUCCURULLO G.*
}

* Istitutio di Ingegneria 'Meccanica, Via Ponte Don Melillo 84084, Fisciano (SA), Italy

\section{Abstrar:t}

In this paper an experimental procedure to determine the thermal conductivities of CFRP thermoplastics laminates sheets is presented. The method is based on Laser heating and Infrared Scanning Radiometer System. The thermal conductivities are estimated by matching the experimental data with the analytical solution, previously derived for the temperature field of heated specimens

\section{Introduction}

Thermal measuring methods are often used as a suitable technique to evaluate a wide range of overall composites characteristics as: thermal properties, porosity, delamination, adhesion quality, density, reinforcement coefficient [1-4]. These last influence mainly the mechanical properties of composites, but also some aspects as the thermal stress evaluation in the laminates [5], which affect the machining accuracy of the workpiece [6]; therefore thermal analysis and evaluation of the thermal characteristics of structural composites [7-11] are needed.

In this work thermal conductivities of unidirectional Carbon Fibre Reinforced Plastic slabs are evaluated by means of an automatic procedure employing integrated Laser heating and Infrared Scanning Radiometer system.

The analytical solution obtained for the temperature field in an anisotropic slab subjected to a confined heating and cooled by forced convection is used to accomplish the data reduction of experimental tests in order to obtain the unknown conductivities.

First experimental results confirm the feasibility of the proposed method as a fast and conomical non-destructive testing.

2. Basic equations and analytical solution.

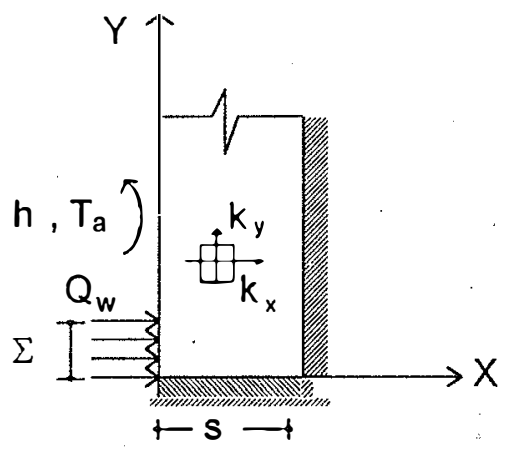

Fig. 1

The slab subjected to a strip heating and cooled by radiative-convective heat transfer to an embiert at uniform temperature is considered, figure 1; in particular the strip heating presents 
a gaussian spatial distribution, $Q=Q_{w, \max } \cdot \exp \left(-Y^{2} / \Sigma^{2}\right)$, characterized by two parameters: $Q_{w, m a x}$, the maximum heat flux incident on the wall and $\Sigma$, the strip heating width. The slab is thermally anisotropic and is modeled as an orthotropic homogeneous solid, characterized by the three principal thermal conductivities which, for the present heating configurations, tum out to be only two, i.e. $k_{x}$ and $k_{y}$.

The energy balance and the boundary conditions for gaussian strip heating can be written in dimensionless form as:

$$
\begin{aligned}
& t_{x x}+t_{y y}=0 \\
& t_{x}(0, y) / B i=-t(0, y)+\exp \left(-y^{2} / \sigma^{2}\right) \\
& t_{x}(1, y)=0 \\
& t_{y}(x, 0)=0 \\
& t_{(x, \infty)}=0
\end{aligned}
$$

where: $x=X / s ; y=Y /\left(s\left(k_{y} / k_{x}\right)^{1 / 2}\right) ; t=\left(T-T_{a}\right) \cdot /\left(\alpha Q_{w, m a x} / h\right) ; \alpha$ is the slab absorbitivity; $h$ is the heat transfer coefficient; $\mathrm{Bi}=\mathrm{h} \cdot \mathrm{s} / \mathrm{k}_{\mathrm{x}}$ is the Biot number; $\sigma=\Sigma /\left(s\left(k_{y} / \mathrm{k}_{\mathrm{x}}\right)^{1 / 2}\right)$ is the dimensionless strip heating width. It has to be noted that the thermal anisotropy of the slab appears as a stretching effect on the reference length normal to the heat flux.

\section{Temperature field}

The problem being linear, the solution for gaussian strip heating can be sought by summing up the system response to a single unit step input:

$$
\begin{aligned}
t(x, y)= & \sum_{n=1}^{\infty} \varphi_{n} \cos \left[\lambda_{n}(1-x)\right] \Phi_{n}(y, \sigma) \\
\Phi_{n}(y, \sigma) /\left[\sigma \sqrt{\pi} / 4 \exp \left(\lambda_{n}{ }^{2} \sigma 2 / 4\right)\right] & =2 \lambda_{n} \cosh \left(\lambda_{n} y\right) \operatorname{erfc}\left(y / \sigma+\lambda_{n} \sigma / 2\right)+ \\
& +\lambda_{n} \exp \left(-\lambda_{n} y\right)\left[\operatorname{erfc}\left(\lambda_{n} \sigma / 2+y / \sigma\right)-\operatorname{erfc}\left(\lambda_{n} \sigma / 2-y / \sigma\right)\right]
\end{aligned}
$$

where $\lambda_{n}$, the eigenvalues related to the Biot number, and the constants $\varphi_{n}$ are given by [7]:

$$
\lambda_{n} \cdot \sin \left(\lambda_{n}\right)=\operatorname{Bi} \cdot \cos \left(\lambda_{n}\right) ; \quad \varphi_{n}=2 \sin \left(\lambda_{n}\right) /\left(\lambda_{n}+\sin \left(\lambda_{n}\right) \cos \left(\lambda_{n}\right)\right)
$$

In order to reduce the number of parameters to be measured and recalling that infrared detection allows to measure surface temperatures, it is convenient to redefine a normalized temperature with respect to the maximum one (i.e. the maximum temperature attained on the exposed surface):

$$
t+=t(x, y) / t(0, y)=t(x, y) / t w(y)=(T(X, Y)-T a) /(T w(0)-T a)=f(x, y, B i, \sigma)
$$

The above dimensionless temperature distribution results to be independent of the heat flux absorbed at wall, i.e. of the slab absorbitivity and the maximum heat flux incident on the wall.

\section{Experimental apparatus.}

The experimental apparatus consists essentially of CFRPIAPC2 sheets $(400 \mathrm{~mm} \times 400 \mathrm{~mm}$ exposed surface and $1-2-3 \mathrm{~mm}$ thickness) made up of epoxy resin matrix in which unidirectional carbon fibres HTS (graphitization temperature $1500^{\circ} \mathrm{C}$ ) are embedded; the fibre volume fraction is about $45 \%$. The test sheets, adiabatic on the bottom, are heated with a $\mathrm{CO}_{2}$ laser source and cooled by a forced air flow at ambient temperature. the related heat transfer 
coefficient is measured by preliminary tests with strip heating on isotropic slabs whose conductivity is known [8].

A computer assisted optical system can realize strip heating by driving the laser spot along a line at $10 \mathrm{~Hz}$. The source presents a gaussian spatial distribution, $Q=Q_{w, \max } \cdot \exp \left(-Y^{2} / \Sigma^{2}\right)$; the maximum heat flux and the strip heating width are controlled via software. The maximum heat flux and the related slab absorbitivity are not measured because the experimental surface temperatures are normalized with respect to their maximum value; while the characteristic length, $\Sigma$, is measured by strip heating too, recalling that for $\mathrm{Bi} \rightarrow \infty$ the normalized temperature profile recovers the normalized heat flux distribution, [9].

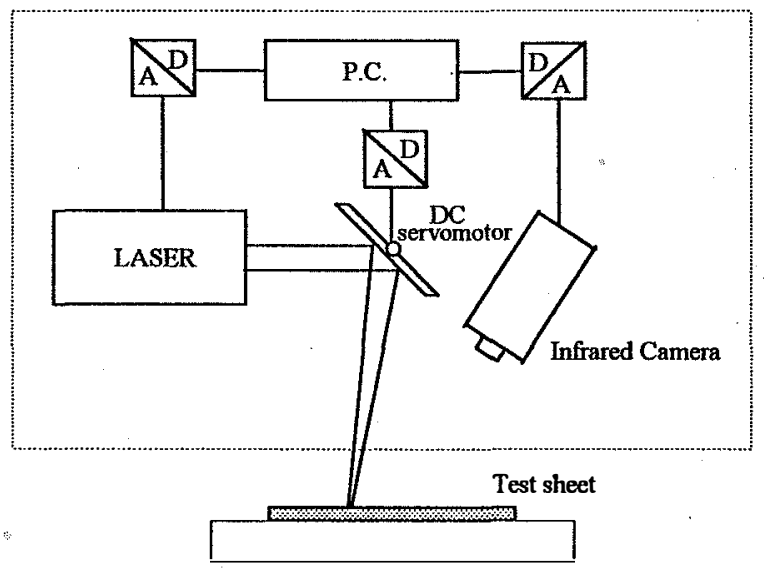

Fig. 2

Surface temperatures are detected by using an Infrared Thermography System (Agema Thermovision $870 / \mathrm{TIC} 8000$ ), with thermal sensitivity of about $0.1^{\circ} \mathrm{C}$ and spatial resolution of about $1 \mathrm{~mm}^{2}$ per pixel.

\section{Experimental procedure}

The CFRPIAPC2 are modeled as orthotropic homogeneous solids, characterized by the three principal conductivities, $k_{\xi}, k_{\eta}$ and $k_{\zeta}$. In particular $k_{\eta}$ is the slab conductivity along the fibres (longitudinal conductivity); $k_{\xi}$ and $k_{\zeta}$ are the conductivities normal to the fibres which, due to the expected uniform fibre distribution, can be assumed to be the same, $k_{\xi}=k \zeta$ (transverse conductivity).

To measure the two unknown thermal conductivities two different heating configurations are used: the first strip heating is parallel to the fibres direction, figure $3 a$, while the second is normal to the fibres, figure $3 b$. This choice allows to decouple the longitudinal and transverse conductivity effects: in fact the parallel strip heating gives directly the transverse slab conductivity (the slab response is isotropic), while the thermal response to the normal strip heating is related to longitudinal and transverse conductivities.

Therefore the experimental procedure provides the following steps:

- for each experiment, two different strip heating are performed: the first one is normal to the fibres direction, the second one is parallel to the fibres

- the surface-ambient temperature difference, detected by means of Infrared Thermography, is normalized with respect to its maximum value 
- the surface temperature integral, $l=\int_{0}^{\infty}\left(T_{w}(Y)-T_{a}\right) /\left(T_{w}(0)-T_{a}\right) \cdot d(Y / s)=f[B i, \sigma]$ is calculated; once $h$ and $\Sigma$ are known, the surface integral results to depend only on the two unknown thermal conductivities of CFRP slab: $l=f\left[k_{x}, k_{y} / k_{x}\right]$
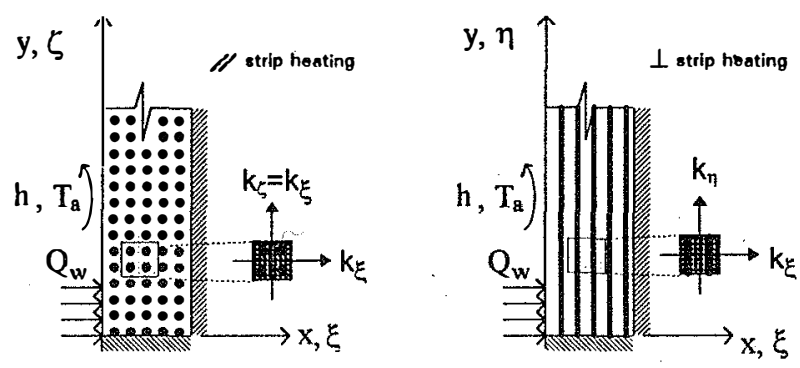

Figs. $3 a$ and $3 b$

- the integral values related to the two strip heating are used to determine the two slab conductivities by comparing them with the analytical solution; in particular, being $I(\|$ heating $)=f\left(k_{\xi}, 1\right]$, the transverse conductivity, $k_{\eta}$, is directly obtained; from $I(\perp$ heating $)=f\left(k_{\xi}\right.$, $\left.k_{\eta} / k_{\xi}\right]$ one has the conductivity ratio, $k_{\eta} / k_{\xi}$.

Tests are performed on CFRPIAPC2 laminae with different thickness, namely $s=1-2-3 m m$, different maximum heat flux incident on the wall and different ambient temperatures. In figure $4 a, b$ the surface temperature plots for normal and parallel strip heating are reported and compared with the analytical solutions.

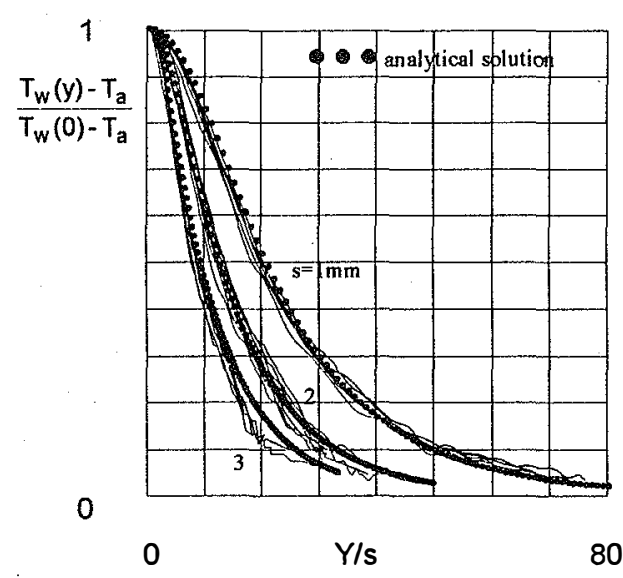

Fig. 4a: normal strip heating

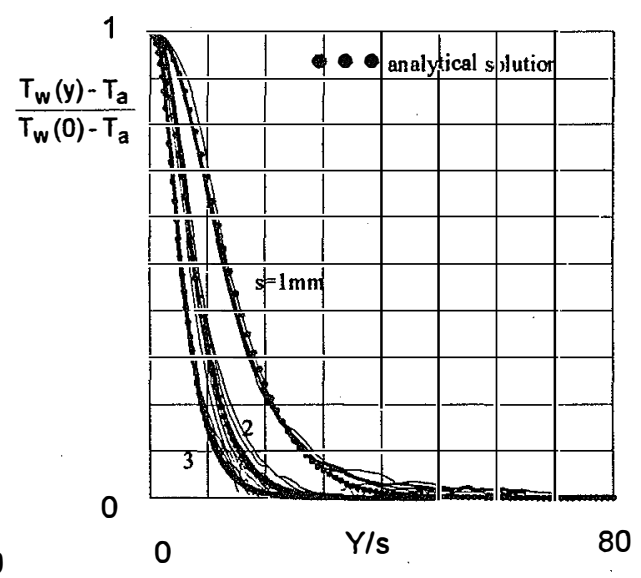

Fig. 4b: parallel strip heating

In the previous fig. the infrared plots related to the same slab thickness result to be quite coincident thus showing that the normalized surface temperature is independent of the maximum heat flux absorbed at wall and the ambient temperature, as expected. 
http://dx.doi.org/10.21611/qirt.1994.013

The measured surface temperature integrals, I $(\|$ heating $)=14.2$ and $I(\perp$ heating $)=25$, lead to $k_{\xi}=0.6 \pm 6 \% \mathrm{~W} / \mathrm{mK}$ and $k_{\eta} / k_{\xi}=7.5 \pm 4 \%$; these values, related to a CFRP with a fibre volume ratio of $45 \%$, are quite similar to those found in literature [10].

\section{Some remarks and conclusions.}

The above measured values of thermal conductivities for CFRPIAPC2 sheets seem to be realistic when compared with values found by [11]: $k_{\xi}=0.72 \mathrm{~W} / \mathrm{mK}$ and $k_{\eta} / k_{\xi}=10$. It has to be noted that in literature different values of thermal conductivities are found: this can be explained by considering that even if the isotropic conductivities of the materials which are composing the CFRP slabs were known, the principal conductivities cannot be directly related to them because they depend on the geometrical disposition of the fibres and on coupling effects at the fibre-matrix interface; furthermore structural imperfection and fibre-packing uncertainties limit the applicability of theoretical models.

Experimental tests carried out using the above procedure enabled the authors to evidence its feasibility; in particular:

- the analytical model seems quite satisfactory to describe the experimental results;

- the experimental protocol together with the analytical model allows a fast-non destructive measurement, an automatic control and low cost set up;

Next developments we are working at are intended toward:

- improvement of present procedure, by considering the time history of surface temperature

- different heating methods, such as moving sources with respect to the slab

\section{REFERENCES}

[1] MATISSE (G.) - Non-destructive testing - Proc. Int. Conf. Advancing with Composites, 1988, Milano, II, pp.437-449.

[2] HARRIS (B.), Phillips (M.G.) - Non-Destructive Evaluation of the Quality and Integrity of Reinforced Plastics - Development in GRP Technology, Applied Science Publishers, 1983.

[3] COMPOSITE DESIGN ENCYCLOPEDIA, Test Methods -vol.6, University of Delaware, 1984.

[4] LAMINE (A.S.), DEGIOVANNI (A.S.), MAILLET (D.) Detection of subsurface defects by a thermai method - Proc. Int. Conf. Advancing with Composites, 1988, Milano, I, pp:313-320.

[5] DI ILIO (A.), TAGLIÁFERRI (V.) - Thermai damage in iaser cutting of (0/90)2s Aramide/Epoxy laminates - Composites, 1989, pp. 115-119

[6] TAGLIAFERRI (V.) DI ILIO (A.) CRIVELLI VISCONTI. (I.)- Laser cutting of fibre reinforced polyester - Composites, 1985, pp. 317-325.

[7] BERARDI (P.G.), CUCCURULLO (G.) - Thermal response evaluation of an anisotropic slab subjected to a confined heating - Proc. XI Congr. U.I.T., 1993, Milano.

[8] BERARDI (P.G.), CUCCURULLO (G.) - Infrared measurements of thermal conductivities for anisotropic slab CFRP thermoplastic laminates - Workshop Infrared Technology and Applications, 1993, Capri.

[9] BERARDI (P.G.), TAGLIAFERRI.(V.), CUCCURULLO (G.) - Thermal Conductivities in Unidirectional CFRP - Advancing with Composites, 1994, Milano.

[10] PILLING (M. W.) - Thermal Conductivity of CFR Composites - Jour. of Material Science, $14: 1326-1338$

[11] MALDAGUE (X. P.V.) - Non destructive evaluation of materials by infrared thermography1993, Springer-Verlag 